\title{
Clés de détermination des Simulies (Diptera, Simuliidae) des Pyrénées
}

\author{
M. Clergue-Gazeaul
}

Mots-Clés : Simuliidae, clés, Pyrénées.

Des clés de détermination (larve, nymphe et imagos) sont données pour les 37 espèces pyrénéennes de Simulies, récoltées par l'auteur entre 400 et 3000 mètres d'altitude.

Keys to species of Simuliidae (Diptera) in the Pyrenees

Keywords : Simuliidae, identification keys, Pyrenees.

Keys for the identification of 37 mountain Simuliidae species in the larval, pupal and adult stages are given. These species were collected between altitudes of 400 and $3000 \mathrm{~m}$ in the Pyrenees.

\section{Introduction}

Depuis les dernières décennies, les Diptères Simuliidae sortent progressivement de leur anonymat. De détermination particulièrement difficile, cette famille très homogène est restée peu utilisée jusqu'à ces derniers temps, malgré l'abondance de ses populations dans les cours d'eau, notamment dans les torrents de montagne ; c'est en effet un des groupes invertébrés les plus rheophiles durant ses stades immatures aquatiques.

Du fait de leur densité très élevée dans nos torrents pyrénéens, et à cause de leur systématique inachevée, les Simulies n'ont été prises que dans leur ensemble, lors des nombreuses études d'impact nécessitées par les activités humaines en montagne (installations de microcentrales, pollution...). Progressivement, avec l'amélioration des possibilités de détermination (Davies 1968, Rivosecchi 1978a, b, Jensen 1984...) ou de classification (Crosskey 1987), ces Diptères ont pu être introduits au niveau espèce dans les études d'ensemble de la faune invertébrés (Giudicelli 1968, Vinçon 1987). C'est pourquoi, il devenait urgent de publier de nouvelles clés françaises, les dernières remontant à Grenier (1953) ; nous avons choisi celles des Simulies d'un massif montagneux français: les Pyrénées.

1. Laboratoire d'Hydrobiologie, U.R.A 695 du CNRS, Université Paul Sabatier, 118, route de Narbonne, 31062 Toulouse Cedex, France.

\section{Méthodes}

Dans les clés, ne sont figurées que les 37 espèces observées par l'auteur en montagne pyrénéenne, à partir de $400 \mathrm{~m}$ d'altitude environ. Quelques éléments non répertoriés, et cités dans des ouvrages plus anciens, n'ont pas été pris en compte, leur présence se révélant douteuse ; ce sont : Metacnephia lesnei, M. pallipes, Simulium (Schoenbaueria) pusillum... Néanmoins, le genre Metacnephia reste introduit dans la clè. Les espèces françaises non observées dans les Pyrénées représentent des éléments du potamon ou des éléments insulaires (Corse).

Au cours de l'établissement des clés, j'ai eu recours, parfois, à celles publiées antérieurement pour l'Italie (Rivosecchi 1978a, b) et la Grande Bretagne (Davies 1968). Chaque espèce ayant été bien décrite dans ces publications et dans Grenier (1953), nous n'en reprenons pas la description et donnons ici simplement des clés, pouvant aider les auteurs qui étudieront la faune benthique de façon détaillée. L'illustration s'est inspirée également des mêmes auteurs, de Knoz (1965), de Jensen (1984), avec des apports personnels.

En premier lieu, sont représentées les clés des genres et des sous-genres, avec des caractères assez vite repérables pour un non spécialiste ; ensuite, chaque sous-genre est repris séparément, pour une détermination jusqu'a l'espèce. Dans la clè des larves, sont données quelques notes écologiques "* " permettant de compléter la connaissance de l'espèce. 
La détermination des genres, sous-genres ou espèces s'accompagne souvent d'une préparation microscopique des individus ; sur les méthodes de dissection, on pourra se référer aux publications de
Rivosecchi (1978 a fig. 3 et 4), en ce qui concerne les larves et les nymphes, et 1967 (fig. 8) du même auteur, pour les adultes.
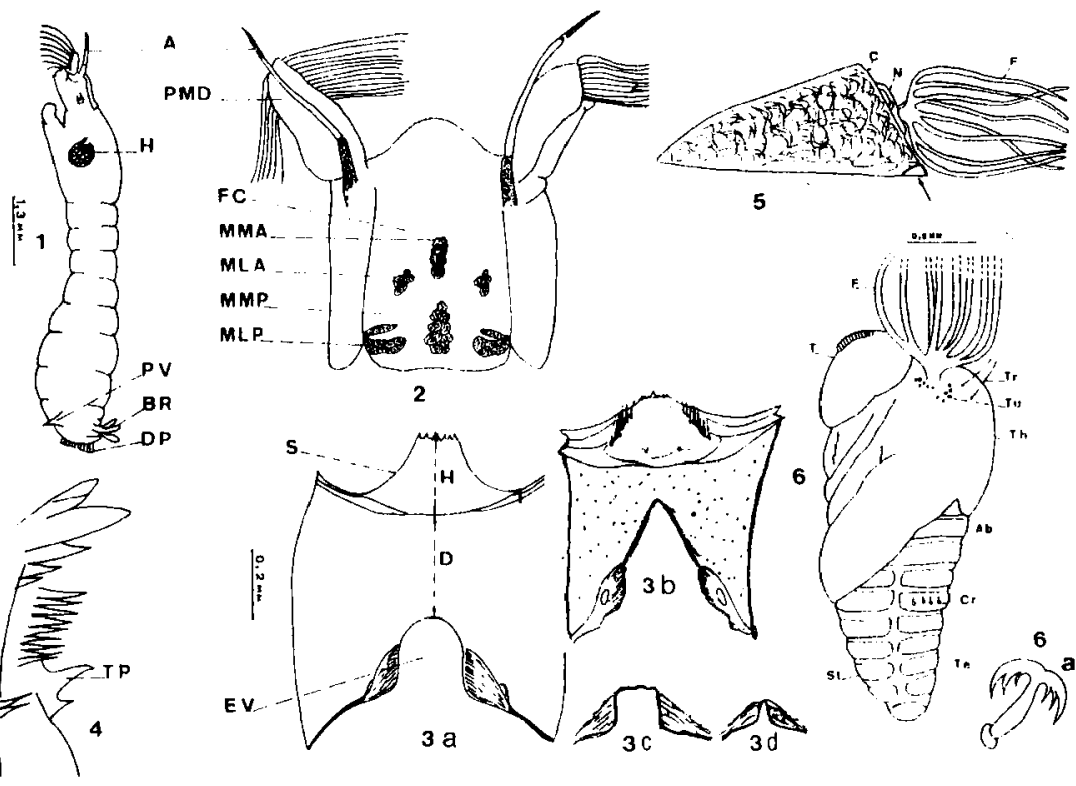

5

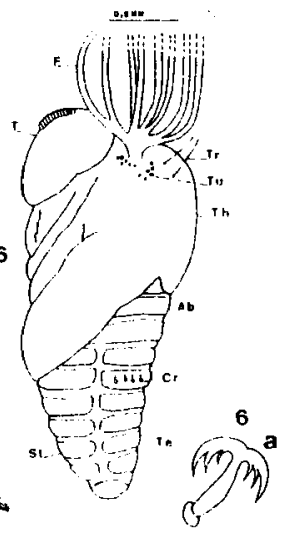

Fig. 1 a 4 . Larve au dernier stade.

1. Vue latérale. A : antenne ; $\mathrm{H}$ : histoblaste des branchies nymphales ; PV : papilles ventrales ; BR : branchies rectales : DP : disque postérieur.

2. Capsule céphalique (Face dorsale). FC : frontoclypeus ; MMA : marque médiane antérieure ; MMP : marque médiane postérieure ; MLA : marques latérales antérieures; MLP ; marques latérales postérieures ; PMD : prémandibule.

3. Capsule céphalique (Face ventrale) de $: S$. (S.) ornatum (3a) ; S : submentum ; EV : échancrure ventrale ; H : hauteur médiane du submentum ; D : distance du bord postérieur du submentum au bord antérieur de l'échancrure ventrale. $3 \mathrm{~b}: S$. (Obuchovia). Echancrure ventrale. 3c:S. (Eusimulium); 3d:S. (Rubzovia).

4. Extrémité antérieure de la mandibule de $S$. (S.) intermedium. TP : processus " tp $"$.

Fig. 5 et 6. Nymphe.

5. Cocon (C) de $S$. (S.) intermedium, avec sa nymphe (N), légèrement sortie afin d'observer les filaments branchiaux (F).

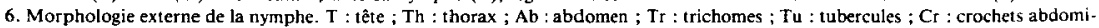
naux : Te : tergite; St : sternite.

6a : crochet en forme d'ancre de la membrane intersegmentaire entre le 8ème et le 9ème sternite abdominal chez le genre Metacnephia. 


\section{Liste faunistique des 37 espèces de Simulies des Pyrénées.}

Classification de Crosskey (1987).

Famille des Simuliidae

Sous-famille des Simuliinae

Tribu des Prosimuliini

Genre Metacnephia

Genre Prosimulium

Sous-Genre Prosimulium

latimucro (End. 1925)

hirtipes (Fr. 1824)

rufipes (Mg 1830)

tomosvaryi (End. 1921)

Tribu des Simuliini

Genre Simulium

Sous-genre Eusimulium

\section{Nevermannia}

Obuchovia

Rubzovia

Simulium

Tetisimulium

gr. venustum

gr. ruficorne

gr. vernum

gr. argenteostriatum

gr. bukowskii

gr. noelleri

gr. ornatum

gr. reptans

gr. tuberosum

gr. variegatum

Wilhelmia angustipes (Edw. 1915)

petricolum (Riv. 1963)

velutinum (S. Abr. 1922)

angustitarse (Ldstr. 1911)

latigonium ( $\mathrm{Rb}$ 1956)

armoricanum D \& Dav. 1961

bertrandi Gr. \& Dor. 1959

brevidens ( $\mathrm{Rb}$ 1956)

carpathicum (Kn. 1961)

carthusiense Gr. \& Dor. 1959

costatum Fr. 1920

cryophilum ( $\mathrm{Rb} 1959$ )

quasidecolletum (Riv. \& Card. 1975)

vernum Macq. 1826

auricoma $\mathrm{Mg} 1818$

lamachi D. \& Dav. 1960

argenteostriatum Str. 1898

hispaniola Gr. \& Bd 1954

degrangei Dor. \& Gr. 1960

noelleri Fr. 1920

intermedium Roub. 1906

ornatum Mg 1818

trifasciatum Curtis 1839

reptans (Lin. 1758)

tuberosum (Ldstr. 1911)

argyreatum $\mathrm{Mg} 1838$

maximum (Kn. 1961)

monticola Fr, 1920

variegatum $\mathrm{Mg} 1818$

xanthinum Edw. 1933

posticatum $\mathrm{Mg} 1838$

bezzii (Corti 1915)

lineatum (Mg 1804) 


\section{Clés analytiques des genres et des sous-genres}

\subsection{Larves}

1. - Bord antérieur du submentum ( $\mathrm{S}: f i g .3$ ) avec dents médiane et latérales nettement plus pointues que les dents intermédiaires; dent médiane à 3 pointes (fig. 15) Prosimulium (Prosimulium)

- Bord antérieur du submentum avec dents médiane et latérales moins apparentes par rapport aux intermédiaires ; dent médiane simple (fig. 15)

2. - Bord supérieur du submenturn très étroit, large comme le $1 / 3$ ou le $1 / 4$ de sa hauteur médiane $(H$ : fig. 3) avec dents à peine visibles (fig. 15)

Metacnephia

- Bord supérieur plus large, avec dents bien visibles (fig. 15) Simulium 3

3. - Extrémité de l'abdomen avec 2 papilles anales (PV : fig. 1) coniques et pointues

- Extrémité de l'abdomen sans papilles anales ou avec papilles à peine visibles

4. - Dents médiane et latérales du submentum plus prononcées que les intermédiaires ; bras du sclérite $X$, au disque postérieur (DP : fig. 1) longs et robustes

- Dents médianes et latérales du submentum moins distinctes des intermédiaires ; bras du sclérite $\mathrm{X}$ petits

Simulium (Nevermannia)

5. - Echancrure ventrale petite (EV : fig. 3), plus ou moins carrée (fig. 3c) Simulium (Eusimulium)

- Echancrure ventrale, très petite, formant 1 encoche en hauteur d' $1 / 2$ fois la largeur (fig. 3d)

Simulium (Rubzovia)

6. - Bord antérieur du submentum droit (fig. 42a) Simulium (Wilhelmia)

- Bord antérieur du submentum flexueux (fig. 41) 7

7. - Submentum très élargi au bord postérieur (fig. 3b) ; disque postérieur avec un grand nombre de rangées de crochets $(150)$

Simulium (Obuchovia)

- Submentum moins large postérieurement (fig. 3a) ; disque postérieur avec un nombre de rangées inférieur ou peu supérieur à 100

8. - Dents latérales du submentum divergentes par rapport à la dent médiane (fig. 41)

Simulium (Tetisimulium)

- Dents latérales du submentum non divergentes par rapport à la dent médiane (fig. 42b)

Simulium (Simulium)

\subsection{Nymphes}

1. - Cocon absent ou de forme non définie, grossièrement tissé (fig. 15) une paire de longues épines sur le 9ème sternite abdominal (St. : fig. 6)

Prosimulium (Prosimulium)

- Cocon présent et de forme bien définie (fig. 15); épines absentes ou très courtes à l'extrémité abdominale

2. - Membrane intersegmentaire située entre le 8ème et le 9ème sternite abdominal pourvue de petits crochets caractéristiques en forme d'ancre (fig. 6a) ; grand nombre de filaments branchiauxMetacnephia

- Absence de crochets en forme d'ancre entre le 8ème et le 9ème sternite abdominal ; nombre de filaments branchiaux $\leqslant 8$ sauf degrangei Simulium 3

3. - Cocon de profil en forme de chaussure à tige (fig. 15)

- Cocon de profil triangulaire en forme de babouche (fig. 15)

4. - Filaments branchiaux très dilatés ; le premier et le dernier forment une collerette autour de la tête de la nymphe (fig. 68)

S. (Wilhelmia)

- Filaments branchiaux tubulaires (fig. 5) ne formant pas de collerette

5. - Cocon avec 1 processus médian et 2 latéraux arrondis au bord antérieur (fig. 64 à 66) S. Simulium gr. argenteostriatum et bukowskit

- Cocon sans processus à la bordure antérieure 6

6. - Cocon en forme de botte, à tige haute; texture compacte (fig. 72)

S. (Obuchovia)

- Cocon en forme de botte, à tige moins haute ; texture lâche et ouvertures à la lisière antéro-Jatérale (fig. 15)

S. (Tetisimulium)

7. - 2-4 filaments respiratoires 8

- 6 à 8 filaments respiratoires S. (Simulium) (autres groupes : noelleri, ornatum, reptans, tuberosum, variegatum, venustum)

8. -2 filaments respiratoires très dilatés (fig. 69) S. (Rubzovia)

-4 filaments respiratoires de forme tubulaire 9

9. - Cocon de structure compacte avec ceinture de renforcement au bord antérieur, sans pointe médiane ; filament supérieut coudé vers le bas (fig. 15)

S. (Eusimulium)

- Cocon de texture pas toujours compacte ; généralement, présence d'un processus médian plus ou moins développé à sa lisière antérieure (fig. 52a); filament dorsal non coudé (fig. 52c)

S. (Nevermannia) 

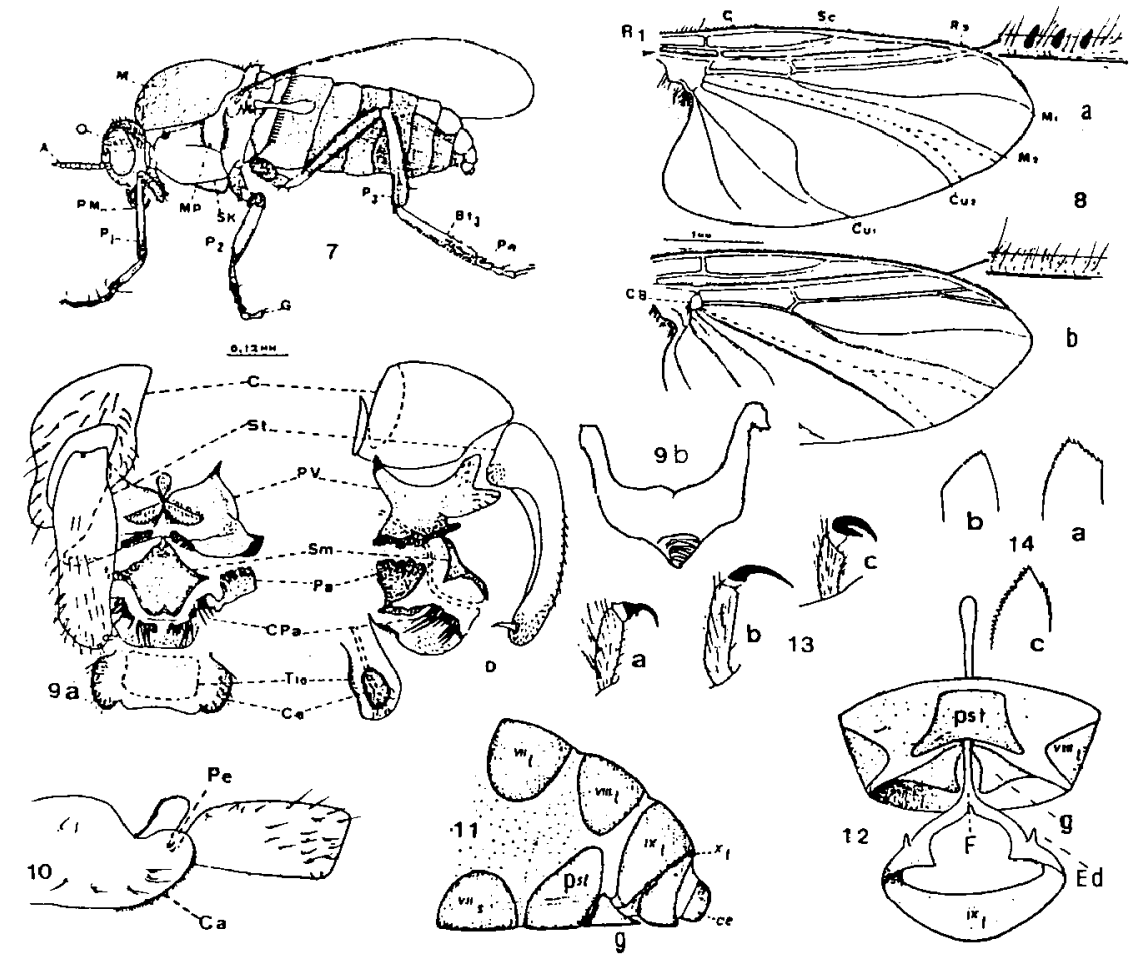

Fig. 7 à 14. Imago.

7. Vue latérale d'une imago femelle (sans nervation alaire). A : Antenne; $O$ : ceil ; PM : palpe maxillaire ; $M$ : mesonotum ; MP : membrane pleurale ; SK : sillon katepisternal ; P1, P2, P3 : pattes, G : griffes ; Bt3 : basitarse de la patte postérieure ; Pe : pedisulcus.

8. Nervation alaire.

Haut : genre Simulium, avec macrotriches sétiformes et spiniformes.

Bas : genre Prosimulium, avec macrotriches uniquement sétiformes.

Rs : secteur radial ; $C$ : costale ; $\mathrm{Cu}$ : cubitale ; $\mathrm{Sc}$ : sous-costale ; $\mathrm{M}$ : médiane ; $\mathrm{CB}$ : cellule basale

9. Genitalia du mâle de : $S$. (S.) argenteostriatum (a). A gauche : vue ventrale ; à droite : vue latèrale et $S$. (S.) degrangei (b). C : coxite ; $\mathrm{St}$ : style ; PV : plaque ventrale ; $\mathrm{Sm}$ : sclérite médian; $\mathrm{Pa}:$ paramères ; T10 : 10ème tergite ; $\mathrm{D}:$ dent apicale; Ce : cerques ; $\mathrm{CPa}$ : crochets des parameres.

10. Extrémité du basitarse postérieur et second segment tarsal de $S$. (N.) cryophilum. Pe : pedisulcus ; C : calcipala.

11. Partie postérieure de l'abdomen de la femelle en vue latérale. $t$ : tergite ; s : sternite ; ce : cerques ; g : gonapophyse ; pst : plaque sternale.

12. Genitalia de la femelle en vue ventrale.

F : furca ; g : gonapophyse ; pst ; plaque sternale ; Ed : épine dorsale.

13. Dernier segment tarsal et griffe tarsale de la femelle. a $: S$. (S.) intermedium; b : $S$. (W.) lineatum ; $c$ : sous-genne Eusimulium.

14. Extrémité distale d'une mandibule. a : $S$. (N.) vernum; b : sous-genre Eusimulium ; c : S. (S.) argenteostriatum. 


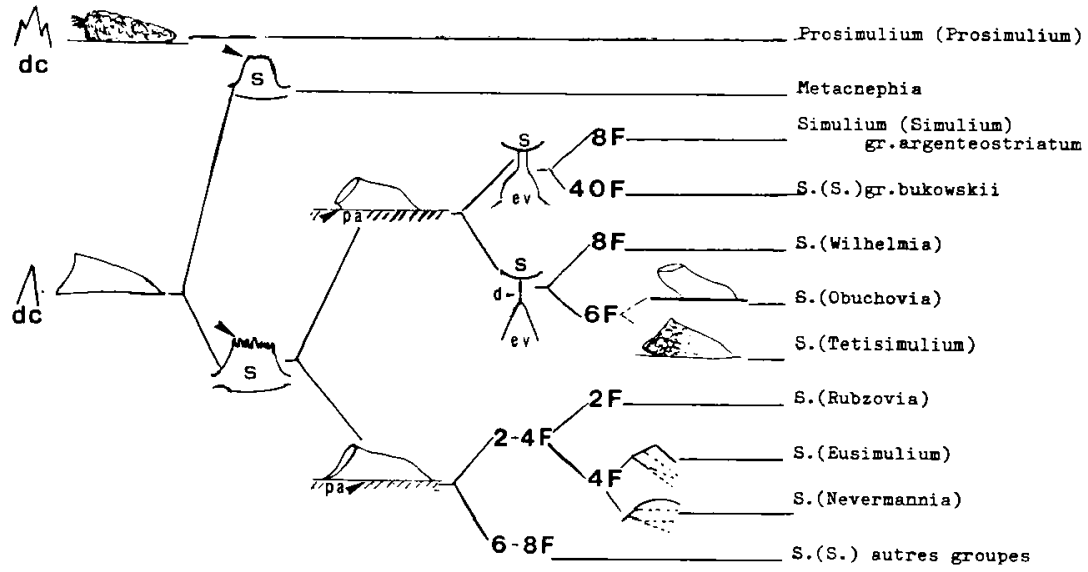

Fig. 15. Schémas de reconnaissance des stades immatures : genres et sous-genres.

$F$ : filaments branchiaux ; $\mathrm{S}$ : submentum ; pa : plan d'attache ; ev : échancrure ventrale ; $d$ : distance du bord postérieur du submentum au bord antérieur de l'échancrure ventrale ; de : dent centrale du submentum.

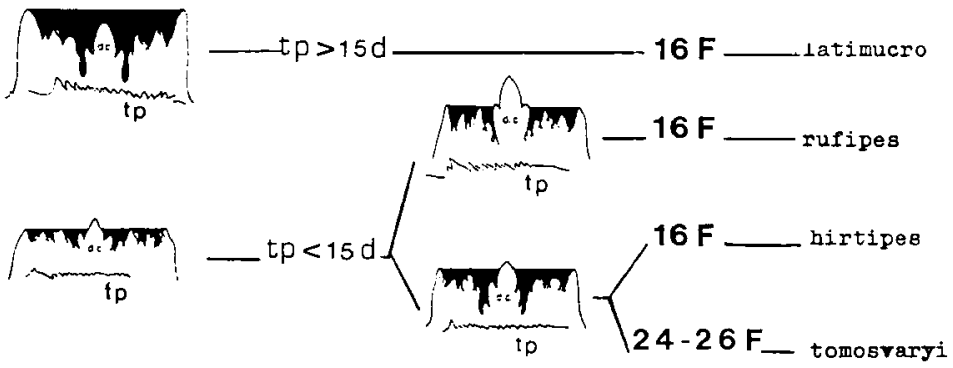

Fig. 16. Schémas de reconnaissance des stades immatures des espèces du sous-genre Prosimulium. F : filaments branchiaux ; $d$ : dents ; dc : dent centrale ; tp : processus tp. 

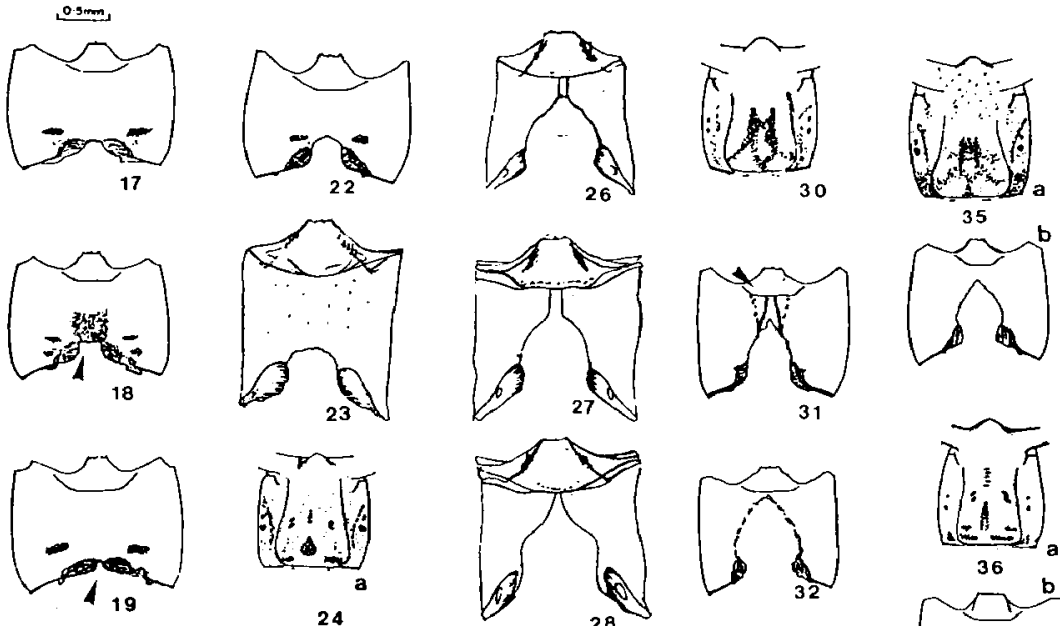

$$
36
$$
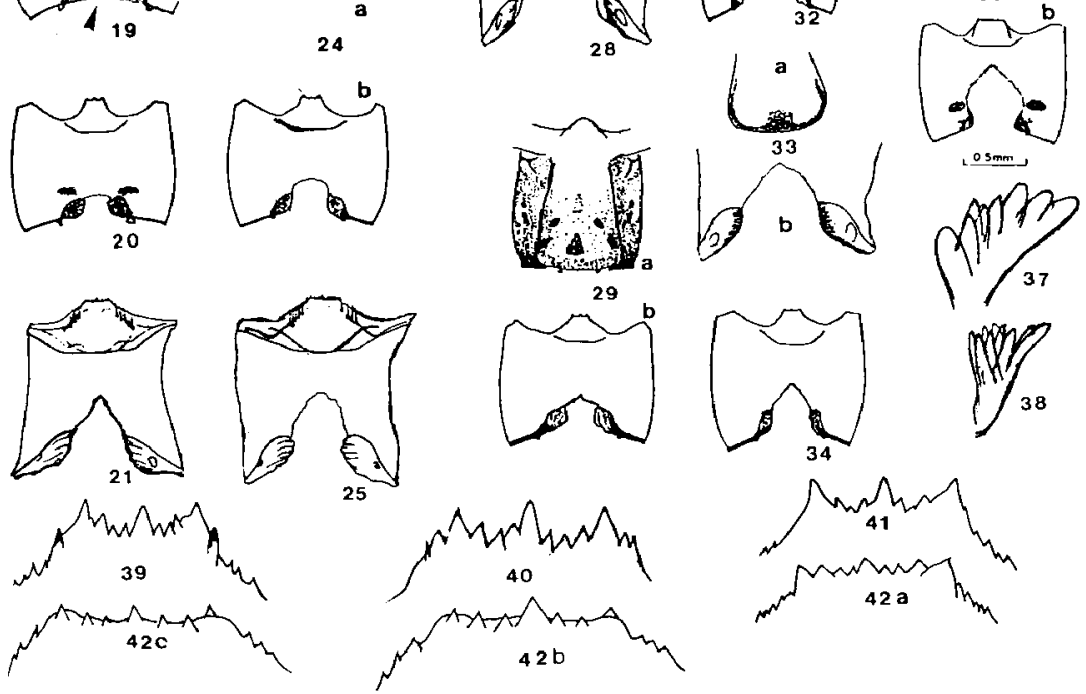

Fig. 17 a 42 . Larves

Echancrure ventrale, $17 ; S$. (N.) angustitarse ; $18 ; S$. (N.) larigonium ; $19 ; S$. (N.) costatum ; $20: S$. (N.) vernum; $21 ; S$. (N.) bertrandi ; 22 : S. (N.) cryophilum; $23: S$. (N.) brevidens; $25: S$. (N.) carthusiense ; $26: S$. (S.) degrangei; $27: S$. (S.) argenteostriatum ; $28: S$. (S.) hispaniola ; $31: S$. (S.) variegatum ; $32: S$. (S.) reptans; $34: S$. (S.) argyreatum.

Frontoclypeus (a) et échancrure ventrale (b). $24: S$. (N.) armoricanum; $29: S$. (S.) trifasciatum ; $33: S$. (S.) maximum ; $35: S$. (S.) noelleri ; $36: S$. (W, linealum.

Frontoclypeus. $30: S .(S$.$) posticatum.$

Branchies rectales. $37: S$. (N.) cryophilum ; $38: S$. (N.) quasidecolletum.

Bord antér ieur du submentum. 39 : sous-genre Eusimulium; $40: S$. (N.) carpathicum ; 41 : sous-genre Tetisimulium ; 42a : sous-genre Wilhelmia ; 42b : $S$. (S.) variegatum; 42c:S. (S.) monticola. 


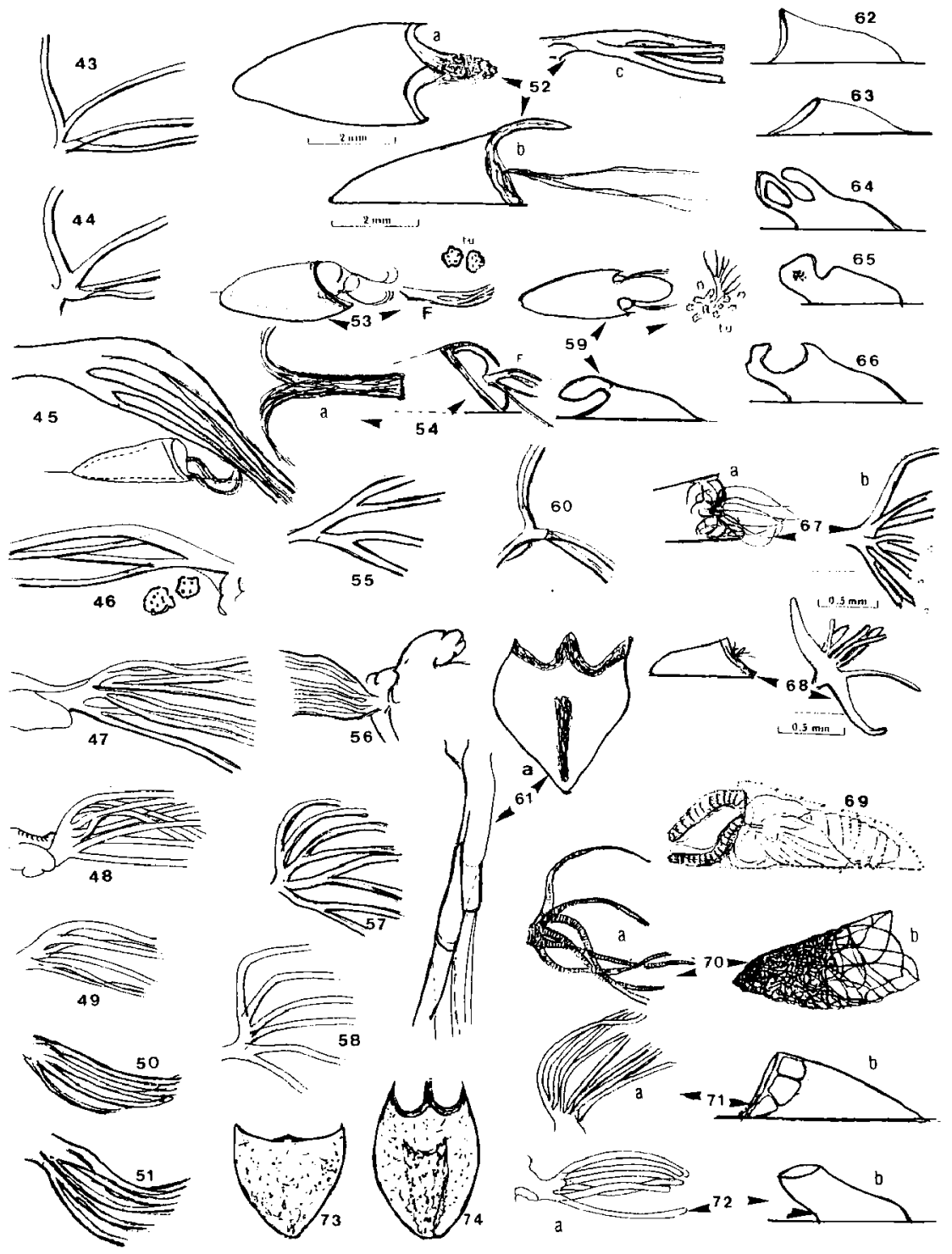




\subsection{Imagos mâles}

Les propositions des numéros 1, 2, 3 et 4 sont valables pour les mâles comme pour les femelles.

1. - Veine costale de l'aile avec seulement des macrotriches en forme de soies, sans spiniformes ; secteur radial bifurqué (fig. $8 \mathrm{~b})$; 2ème segment tarsal sans pedisulcus ; basitarse IJl (Bt3) sans calcipala mais avec une extension épineuse au coin dis. tal inférieur ; grandes espèces

Prosimulium (Prosimulium)

- Veine costale de l'aile avec macrotriches de 2 types : spiniformes et en forme de soies; secteur radial simple (RS, fig. 8a) ; coin distal postérieur du basitarse III avec calcipala $(f i g .10)$

\section{2}

2. - Pedisulcus absent (ou très peu distinct) ; calcipala présent

Metacnephia

- Pedisulcus (fig. 10) toujours présent ; calcipala (Ca : fig. 10) toujours présent sur basitarse III (fig. 10)

Simulium 3

3. - Section basale de la veine radiale (R I) avec macrotriches à sa surface supérieure (fig. 8a)

4

- Section basale de la veine radiale nue à sa surface supérieure (clé des mâles) (clé des fernelles)

4. - Membrane pleurale (MP : fig. 7) avec nombreuses soies ; basitarse III étroit, environ la moitié de la largeur maximum du tibia

S. (Wilhelmia)

- Membrane pleurale nue ; basitarse III élargi, au plus aussi large que la largeur maximum du tibia

5. - Style (St : fig. 9) de même longueur ou seulement un peu plus court que le coxite ; plaque ventrale laminaire, quadrangulaire en vue frontale avec le bord dorsal du corps soudé au bras sur toute la longueur (fig. 80)

S. (Nevermannia)

- Style nettement plus court que la coxite ........6 6
6. - Plaque ventrale en vue frontale, avec corps mince et triangulaire plus court et plus étroit que les bras (fig. 79)

S. (Eusimulium)

- Plaque ventrale laminaire, large et transversale avec carène médiane longue pourvue de soies; bras petits et forts dirigés vers l'avant (fig. 90)

\section{S. (Rubzovia)}

7. - Style très long. 4 fois plus long que la hauteur du coxite (fig. 108) S. (Obuchovia)

- Style moins long. I fois $1 / 2$ à 2 fois la hauteur du coxite (fig. 107)

8. - Basitarse I très dilaté et aplati, 4 à 5 fois plus long que large ; basitarse III élargi $S$. (Simulium)

- Basitarse I non dilaté, 6-8 fois plus long que large ; basitarse III non dilaté, 5 fois plus long que large

S. (Tetisimulium)

\subsection{Imagos femelles}

Pour les propositions 1, 2, 3 et 4 se reporter au mâle.

5. - Mandibule toujours dentelée d'un seul côté (fig. 14a) ; gonapophyses (G : fig. 12 et 11 ) formant 2 lobes pointus

S. (Eusimulium)

- Mandibule dentelée ou non des deux côtés ; gonapophyses formant 2 lobes non pointus (fig. 120)

S. (Nevermannia)

S. (Rubzaria)

6. - Basitarse I non dilaté, environ 7 fois plus long que large ; mesonotum ( $\mathrm{M}:$ fig. 7 ) à coloration de fond gris avec 3 stries noires $\quad S$. (Tetisimwlium)

- Basitarse I dilaté 4 à 5 fois plus long que large ; mesonotum noir avec ou sans taches argent ées (sauf xanthinum)

7. - Gonapophyses pointues et longues (fig. 123).

S. (Obuchovia)

- Gonapophyses triangulaires ou arrondies, non pointues postérieurement (fig. 124)S. (Simulium)

Fig. 43 à 74. Nymphes.

Partie basale des filaments respiratoires. $43: S$. (N.) angustilarse; $44: S$. (N.) latigonium ; $46: S$. (N.) carthusienne ; $47 ; S$. (S.) argenteostriatum ; $48: S$. (S.) hispaniola ; $49 ; S$. (S.) argyreatum ; $50: S$. (S.) monticola ; $51: S$. (S.) maximum ; $55: S$. (N.) costatum ; $56: S$. (S.) variegatum, avec patagium ; $57 ; S$. (S.) (rifasciatum; $58: S$. (T.) bezzii ; $60: S$. (N.) carpathicum.

Forme de cocon nymphal. $59: S$. (N.) bertrandi ; $62: S$. (S.) argyreatum ; $63: S$. (S.) monticola ou maximum ; $64: S .(S$.$) argenteos-$ triatum ; 65:S. (S.) hispaniola ; $66: S$. (S.) degrangei; $73: S$. (N.) angustitarse ; $74: S$. (N.) latigonium.

Filaments respiratoires et forme du cocon. $45: S$. (N.) brevidens $; 52: S$. (N.) cryophilum $; 53: S$. (N.) quasidecolletum ; $54: S$. (N.) vernum ; $61:$ S. (N.) armoricanum ; $67: S$. (S.) noelleri ; $68: S$. (W.) lineatum ; $70: S$. (S.) anthinum; $71 ; S$. (S.) reptans ; 72 : $S$. (O.) auricoma.

Nymphe et cocon. 69 : S. (R.) Iamachi.

$\mathrm{Tu}$ : tubercules thoraciques. 

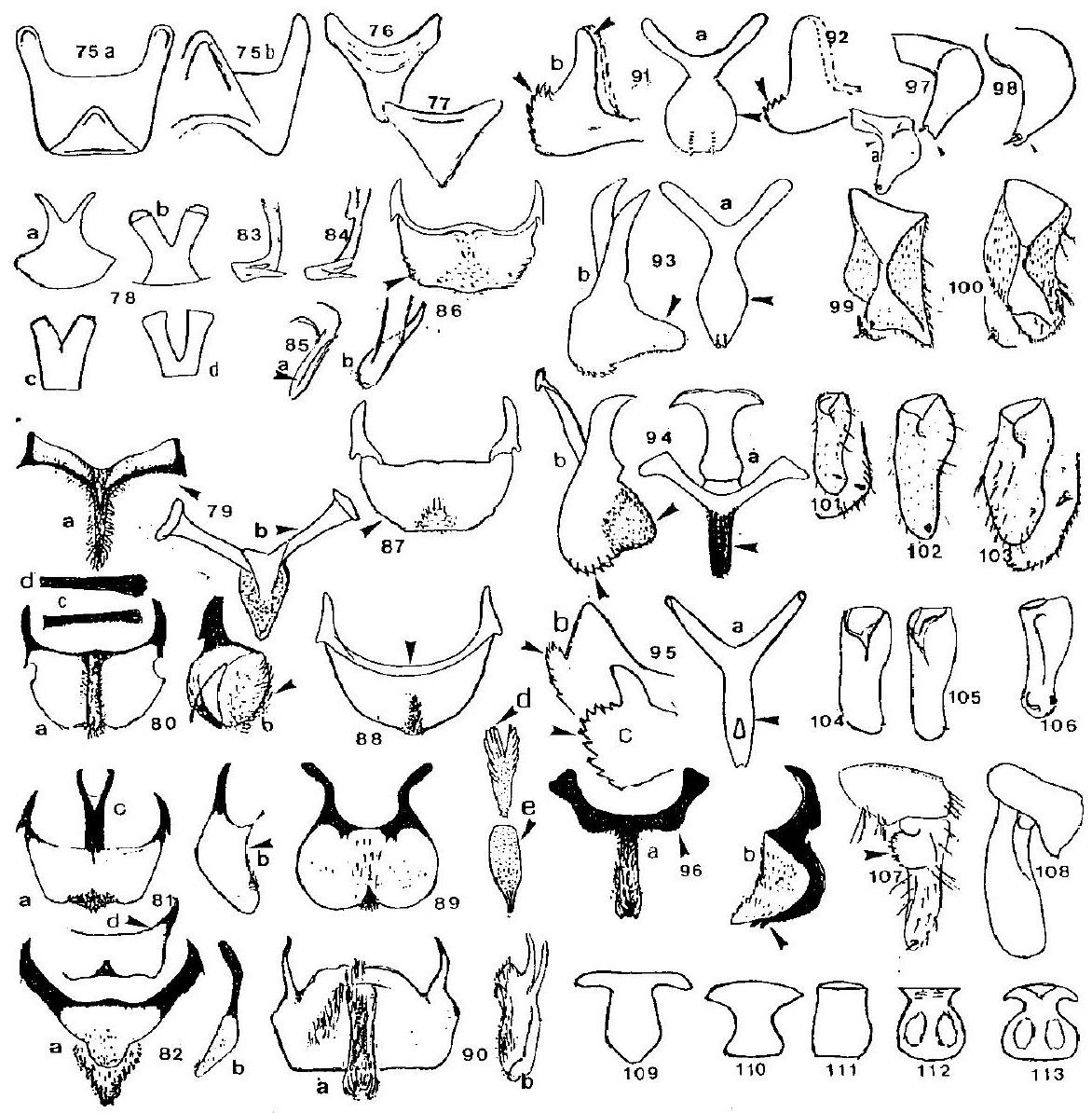


\section{Clés analytiques des espèces pyrénéennes}

\subsection{Sous-genre Prosimulium}

4.1.1. Larves (fig. 16)

1. - Pointes des dents latérales et intermédiaires du submentum disposées en 2 lignes divergentes (en ( $V$ »); processus tp de la mandibule avec plus de 15 dents

latimucro

* Haute altitude ; déversoirs de lacs ou de glaciers

- Pointes des dents latérales et intermédiaires du submentum disposées sur une même ligne horizontale ; processus tp de la mandibule avec moins de 15 dents

2. - Les pointes de dents latérales de la dent trifide centrale du bord antérieur du submentum sont à peu près sur la même ligne que les pointes des dents latérales

rufipes

* Orophile : 1000-2000 m

- Au bord antérieur du submentum, les pointes des dents latérales de la dent trifide centrale sont très en dessous de la ligne teliant les pointes des dents latérales du submentum

3. - Processus tp de la mandibule avec dents bien marquées, particulièrement la première dent antérieure ; soies principales des baguettes du prémandibule (PMD : fig. 2) plus longues que le diamètre de la baguette

tomosvaryi

* La moins sténotherme ; 0-1500 m

- Processus tp de la mandibule avec dents peu marquées, particulièrement la lère ; soies principales des baguet tes du prémandibule de longueur égale au diamètre de la baguette

* Orophile : moyenne altitude.
4.1.2. Nymphes (fig. 16)

1. - Organe respiratoire avec 20-26 filaments tomosvaryi

- Organe respiratoire avec 16 filaments

2. - Les 16 filaments sont groupés hirtipes

- Les 16 filaments sont plus ou moins divergents

3. - Troncs basaux allongés $(0,6-0,7 \mathrm{~mm})$ latimucro

- Troncs basaux courts $(0,3-0,4 \mathrm{~mm}) \quad$ rufipes

4.1.3. Imagos mâles

1. - Plaque ventrale, en vue frontale, avec corps aussi large que haut (fig. 75a) ; sclérite médian en $\mathrm{Y}$ (fig. 78b)

tomosvaryi

- Plaque ventrale (vue frontale) plus large que haute (fig. 75b)

2. - Coxite plus large que haut ; style court, conique ; bras du sclérite médian dilatés distalement (fig. 78d)

latimucro

- Coxite plus haut que large ; style allongé ; bras du sclérite médian non dilatés distalement

(fig. 78a)

3. - Corps de la plaque ventrale, en vue latérale, non pointu en avant ; vue ventrale en forme de triangle équilatéral (fig. 77) ; bras du sclérite médian larges et pell divergents (fig. $78 \mathrm{c}$ ) hirtipes

- Corps de la plaque ventrale en vue latérale, très pointu ; vue ventrale, en forme de triangle isocèle (fig. 76) ; sclérite médian avec bras minces et bien divergents (fig. 78a) rufipes

Fig. 75 à 113. Imagos mäles.

Plaque ventrale en vue frontale. $75 \mathrm{a}: P .(P$.$) tomosvaryi; 75 \mathrm{~b}: P .(P$.$) rofipes; 86: S .(N$.$) carthusiense ; 87: S .(N$.$) quasidecolle-$ tum ; $88: S$. (N.) bertrandi; 79a : angustipes; $79 \mathrm{~b}:$ velutinum.

Plaque vent rale en vue ventrale. $76: P$. $P$.) rufipes $: 77: P$. (P.) hirtipes.

Plaque ventrale en vue latérale. $92: S$. (S.) intermedium.

Plaque ventrale en vue frontale (a) et latérale (b) ou en vue frontale. $82: S$. (W.) lineatum; $89: S$. (O.) auricoma ; $91: S$. (S.) ornatum( ; $93: S$. (S.) trifasciatum; $95: S$. (S.) variegatum; $96: S$. (S.) reptans; $81 \mathrm{~d}: S$. (N.) armoricanum.

Plaque vent rale en vue frontale (a) en vue latérale (b) et sclérite médian. $80: S$. (N.) latigonium : $81: S$. (N.) vernum; $90: S$. (R.) lamachi; 94 : S. (S.) noelleri.

Crochets des paramères. $83: S$. (S.) angustitarse; $84: S$. (N.) latigonium; 85a : S. (N.) vernum.

Sclérite médian. $78: P$. (P.) : a : rufipes ; b : lomosvaryi, c : hirtipes, d : latimucro ; $80: S$. (N.) : c : latigonium, d : angustitarse ; $95 \mathrm{~d}: S$. (S.) variegatum : $95 \mathrm{e}: S$. (S.) xanthimum.

Style. $97: S$. (E.) petricolum; $98:$ S. (E.) angustipes; $99: S$. (N.) vernum ; $100: S$. (N.) carpathicum; $101: S$. (S.) argyreatum ; $102: S$. (S.) maximum; $103:$ S. (S.) monticola; $104: S$. (S.) ornatum; $105: S$. (S.) intermedium; $106: S$. (T.) bezzii ; 97 a : S. (E.) velutinum.

Style et coxite. $107: S$. (S.) tuberosum; $108: S$. (O.) auricoma.

Forme du Xème sternite. $109: S$. (N.) brevidens : $110: S$. (N.) cryophilum ; $111:$ S. (N.) carpathicum : $112: S$. (N.) carthusiense ; $113: S$. (N.) quasidecolletum. 

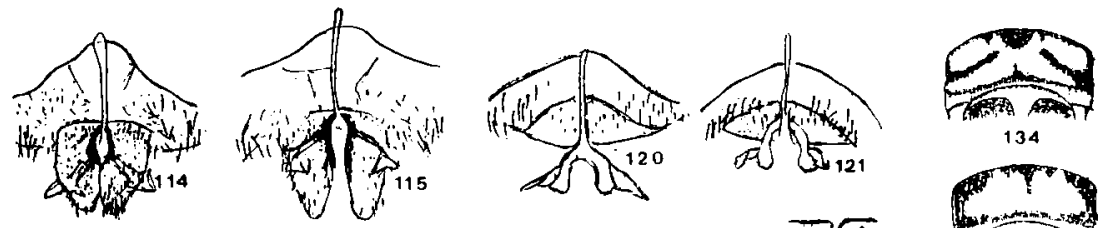

134
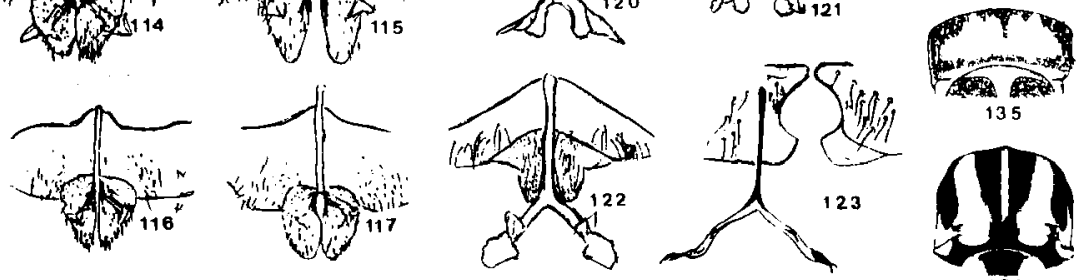

135
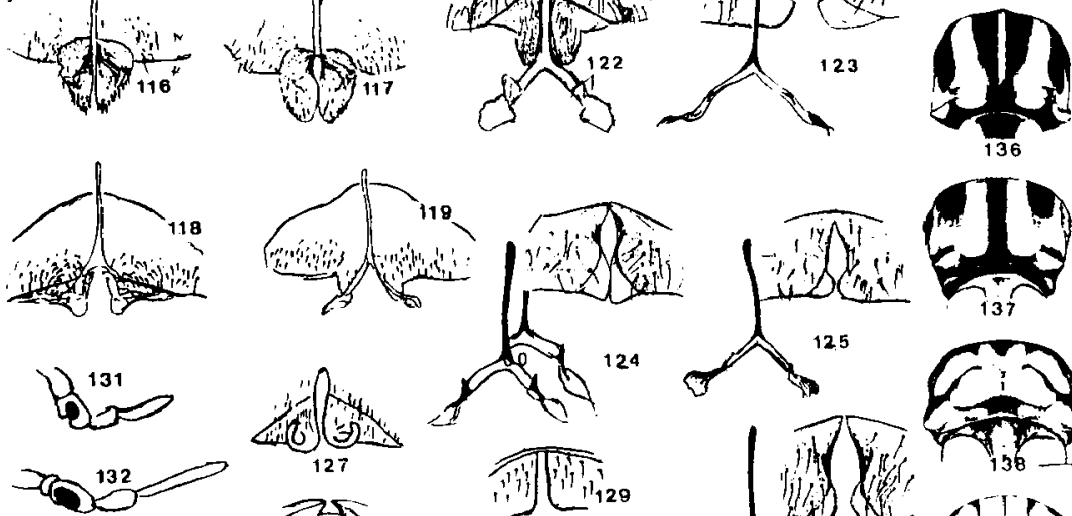

127
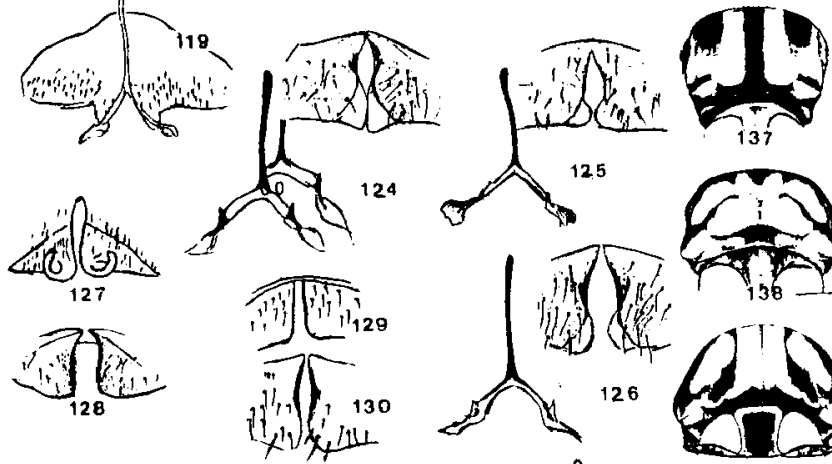

137
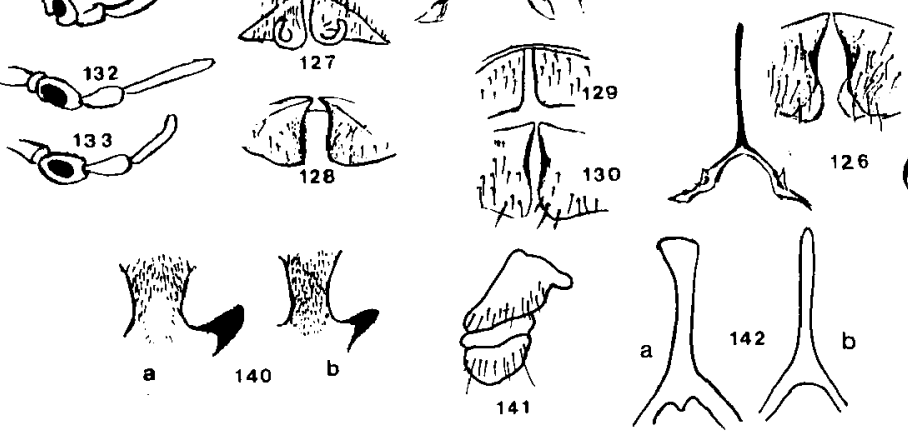

Fig. 114 à 139. Imagos femelles.

Gonapophyses et furca de la femelle. $114: P$. (P.) tomosvaryi $; 115: P$. (P.) latimucro; $116: P$. (P.) hirtipes; $117: P$. (P.) rufipes ; $118: S$. (N.) angustitarse; $119: S$. (N.) latigonium; $120: S$. (N.) brevidens; $121: S$. (N.) carpathicum ; $122: S$. (O.) auricoma: 123 : S. (S.) ornatum ; $124: S$. (S.) argyreatum; 125 : S. (S.) monticola ; $126: S$. (S.) maximum.

Gonapophyses de la femelle. $127: S$. (W.) lineatum; $128: S$. (S.) reptans; $129: S$. (S.) tuberosum; $130: S$. (S.) noelleri.

Pajpe maxillaire et crypte sensorielle. $131: S$. (N.) vermum; $132 ; S$. (N.) brevidens; $133 ; S$. (N.) cryophilum.

Mesonotum en vue frontale. $134: S$. (S.) ornatum; $135: S$. (S.) reptans; $136: S$. (S.) hispaniola; $137: S$. (T.) bezzii ; $138: S$. (S.) variegatum ; $139: S$. (S.) monticola.

Front de la femelle de : $140 \mathrm{a}: S$. (E.) velutinum; $140 \mathrm{~b}: S$. (E.) angustipes.

Cerques. $141: S$. (N.) vernum.

Furca. 142a : $S$. (N.) vernum; 142b : $S$. (N.) bertrandi. 


\subsubsection{Imagos femelles}

1. - Gonapophyses grandes, plus longues que la hauteur du 8ème sternite

- Gonapophyses petites, égales ou inférieures à la hauteur du 8ème sternite 3

2. - Tige médiane de la furca longue, plus du double de chaque branche latérale; gonapophyses avec bords externes courbes, bords internes flexueux se rejoignant sur la ligne médiane

(fig. 114)

tomosvaryi

- Tige médiane de la furca 1 fois $1 / 2$ à 2 fois maximum la longueur de chaque branche laterale: gonapophyses avec bords externes droits, bords internes flexueux ne se rejoignant pas sur la ligne médiane (fig. 115)

latimucro

3. - Gonapophyses triangulaires, pointues distalement (fig. 116); pattes sombres hirtipes

- Gonapophyses arrondies, non pointues distalement (fig. 117); pattes jaune ocre

rufipes

\subsection{Sous-genre Eusimulium}

\subsubsection{Larves}

1. - Couleur de fond du fronto-clypeus sombre petricolum

* Surfaces hygropétriques; sources en zone semiaride.

- Frontoclypeus avec taches sombres sur fond clair sans aucune ombre

2. - Echancrure ventrale peu profonde, carrée avec bord droit ou très légèrement dentelé velutinum

* Pjémont

- Echancure ventrale plus profonde, ogivale avec bord dentelé

angustipes

* Biotope humide ; ruisseaux froids riches en substances nutritives.

\subsubsection{Nymphes}

Il est extrêmement difficile de départager ces 3 espèces à l'état nymphal ; se reporter à la clé de la larve ou du mâle.

\subsubsection{Imagos mâles}

1. - Style avec bord interne formant un angle droit (fig. 97a) ; plaque ventrale, en vue frontale, plutôt mince (fig. 79b)

2

- Style avec bord interne formant un angle obtu (fig. 98) et extrémité pointue ; plaque ventrale massive en vue frontale avec soies longues et robustes (fig. 79a)

angustipes

2. - Style petit, à extrémité arrondie ( $/ i g .97 \mathrm{a})$ velutinum

- Style plus grand à extrémité carrée (fig. 97) petricolum

\subsubsection{Imagos femelles}

1. - Front large, un peu plus haut que la largeur minimale; aire médiane nue; grand angle frontooculaire (fig. 140a)

velutinum

- Front étroit ; 1 fois $1 / 2,2$ fois plus haut que la largeur minimale ; angle fronto-oculaire étroit (fig. 140b)

2. - Basitarse 1 mince ( 7 fois plus long que large) et moins long que le double de l'article suivant ; basitarse III dilaté et environ 5 fois plus long que large petricolum

- Basitarse I moins mince ( 6 fois plus long que large) et environ 2 fois la longueur de l'article suivant ; basitarse III moins dilaté, environ 6 à 8 fois plus long que large

angustipes

\subsection{Sous-genre Nevermannia}

\subsubsection{Larves}

1. - Frontoclypeus (FC: fig. 2) à fond clair ; échancrure ventrale plus ou moins distincte ; branchies rectales simples ( $\mathrm{Br}:$ fig. 1)

groupe ruficorne 2

- Frontoclypeus à fond plus ou moins sombre ; échancrure ventrale bien visible; branchies rectales ramifices (fig. 38) ..... groupe vernurn 3

2. - Echancrure ventrale très petite (fig. 17) ; aire antérieure de l'échancrure non pigmentée ; tache postéromediane (MMP : fig. 2) du frontocypeus en forme de triangle isocèle à base élargie ; bord antérieur du submentum avec 2 dents intermédiaires entre les latérales et la médiane angustitarse

* Rare; petites rivières à cours lent.

- Echancrure ventrale plus grande ; aire antérieure de l'échancrure ventrale pigmentée (fig. 18); tache postéromédiane du frontoclypeus en forme de stries allongées et étroites ; 3 dents intermédiaires entre les latérales et la médiane au bord antérieur du submentum latigonium

* Petits cours d'eau lents ; crénon.

3. - Echancrure ventrale étroite et peu profonde ; distance au bord postérieur du submentum égale ou supérieure à 2 fois la hauteur du submentum (fig. 19)

- Echancrure ventrale plus profonde et plus large (fig. 21)

4. - Echancrure ventrale très petite, formant une légère entaille au bord postérieur ventral de la capsule céphalique (fig. 19)

costatum

- Echancrure ventrale plus profonde et plus large, (fig. 20), de forme carrée vernum

* Petits cours d'eau; crénon ou rhithron. 
5. - Tache postéromédiane du frontoclypeus en triangle équilatéral presque aussi large que haut (fig. $24 a)$; processus “ tp » de la mandibule avec une seule dent bien visible et une dent subsidiaire unique minuscule

armoricanum

* Rare ; petits cours d'eau de montagne ; piémont.

- Tache postéromédiane du frontoclypeus en triangle isocèle, souvent étroite : processus " tp » avec une dent principale et plusieurs petites dents secondaires bien visibles

6. - Dent médiane du bord antérieur du submentum nettement supérieure en longueur aux dents intermédiaires (fig. 40)

carpathicum

* Orophile ; ruisseaux de source

- Dent médiane du bord antérieur du submentum environ de même dimension que les dents intermédiaires ou un peu plus longue

A partir de la proposition 7 , il existe quelques difficultés dans la détermination des larves ; il est prudent de com. pléter la détermination à l'aide de la clé des nymphes.

7. - Distance entre le point maximum de profondeur de l'échancrure ventrale et le bord postérieur du submentum, inférieure à la hauteur médiane du submentum

- Distance entre le point maximum de profondeur de l'échancrure ventrale et le bord postérieur du submentum, égale ou légèrement supérieure à la hauteur médiane du submentum ............ 9

8. - Echancrure ventrale en angle aigu (fig. 21) ; frontoclypeus sombre avec impressions indistinctes ; processus tp avec 7-8 dents

bertrandi

* Ruisseaux de montagne ; $700-2200 \mathrm{~m}$.

- Echancrure ventrale ogivale (fig. 25) ; taches du frontoclypeus bien distinctes; processus tp avec 3-4 dents carthusiense

* Torrents à fond pierreux et eau pure : $300-2500 \mathrm{~m}$.

9. - Distance entre échancrure ventrale et lisière postérieure du submentum supérieure à la hauteur médiane du submentum (fig. 23) brevidens

* Stenotherme ; eau froide et pure des glaciers ; nuisseaux en forêt.

- Distance entre l'échancrure ventrale et la lisière postérieure du submentum égale à la hauteur médiane du submentum

10. - Digitations secondaires des branchies rectales minces : longues comme 6-10 fois le diamètre de chaque digitation (fig. 38) ; échancrure ventrale ogivale

quasidecolletum

* Rare dans les Pyrénées; fond pierreux.

- Digitations secondaires, environ une fois, une fois et demi plus longues que le diamètre de chaque digitation (fig. 37) ; échancrure ventrale arrondie et bien visible (fig. 22)

cryophilum
* Largement répandue ; grande amplitude altitudinale; ruisseaux de source, ruisseaux et rivières de montagne et de piémont.

\subsubsection{Nymphes}

1. - Filament le plus dorsal des 4 filaments respiratoires très éloigné des 3 autres

groupe ruticorne 2

- Filaments respiratoires plus ou moins accolés les uns aux autres

groupe vernum 3

2. - Diamètre de la paire de filaments dorsaux à peu près égal à celui des filaments ventraux (fig. 43) ; cocon à texture lâche avec processus médian à peine formé (fig. 73)

angustitarse

- Diamètre de la paire de filaments dorsaux, à la base environ le double de celle des filaments ventraux (fig. 44); cocon à structure compacte avec processus médian antérieur très pointu (fig. 74)

latigonium

3. - Cocon sans pointe médiane au bord antérieur ( $f i g$. 53)

- Cocon avec pointe médiane au bord antérieur (fig. 52a)

4. - Filaments branchiaux étroitement accolés (fig. 53F) quasidecolletum

- Filaments branchiaux écartés les uns des autres (fig. 55) ; bords du cocon bien épaissis ; grande espèce

costatum

5. - Cocon tissé de manière épaisse avec fils à structure granuleuse; bords latéraux, antérieur et bande longitudinale médiane, épaissis (fig. 61a) armoricanum

- Cocon tissé bien plus finement avec fils à tissage uniforme

6. - Les 4 filaments respiratoires accolés 2 à 2 , les 2 couples nettement divergents (fig. 60) carpathicum

- Les 4 filaments rapprochés entre eux, la paire dorsale plus ou moins divergente par rapport à la ventrale

7. - Cocon avec pointe médiane antérieure longue et étroite à la base (fig. 54a)

- Cocon avec pointe médiane antérieure plus courte et élargie à la base (fig. 52a)

8. - Tégument céphalique et thoracique avec tubercules lisses; cocon avec pointe médiane antérjeure étroite sur toute sa longueur vernum

- Tégument céphalique et thoracique avec tubercules rugueux par la présence de grosses verrues ; pointe médiane importante en forme de cuillère ; trichomes ramifiés (fig. 59)

bertrandi 
9. - Tégument céphalique et thoracique avec tubercules à structure rugueuse carthusiense

- Tégument céphalique et thoracique avec tubercules lisses

10. - Filaments respiratoires très longs et minces même à la base (fig. $52 \mathrm{~b}$ et c) cryophilum

- Filaments respiratoires plus courts et dilatés à la base (fig. 45)

brevidens

4.3.3. Imagos mâles

1. - Plaque ventrale en vue latérale, avec bord antérieur nettement convexe (fig. 80b) ; crochets des paramères courts ; sclérite médian non bifide distalement (fig. 80a) gr. ruficorne ........ 2

- Plaque ventrale en vue latérale, avec bord antérieur concave, pointue ventralement en vue latérale $(f i g .81 \mathrm{~b})$; crochets des paramères plutôt longs ; sclérite médian bifide distalement (fig. 81c)

$$
\text { gr. vernum } 3
$$

2. -2 crochets aux paramères, le plus grand recouvrant presque intégralement le plus petit (fig. 83 ) ; sclérite médian arrondi distalement (fig. $80 \mathrm{~d}$ ) angustitarse

- 2 crochets aux paramères, le plus grand ne recouvre pas le plus petit (fig. 84) ; sclérite médian non arrondi distalement (fig. 80e) latigonium

3. - Les deux portions de l'unique crochet du paramère accolées sur toute la longueur (fig. 85a) ......4

- Les 2 portions de l'unique crochet du paramère ne sont pas accolées sur toute la longueur (fig. 85b)

4. - Plaque ventrale trapézoidale en vue frontale ( $f i g$. 81 a) ; style très dilaté vers la base, largeur maximale environ les $3 / 4$ de la hauteur (fig. 99) ; basitarse III pas très dilaté, environ 4 fois et demi plus long que large

vernum

- Plaque ventrale rectangulaire en vue frontale fig. $81 \mathrm{~d}$ ) ; style moins dilaté et plus petit ; basitarse III très dilaté : longueur inférieure à 4 fois la largeur

armoricanum

5. - Bord antérieur de la plaque ventrale concave (fig. 88)

bertrandi

- Bord antérieur de la plaque ventrale convexe en son milieu (fig. 86)

6. - Sternite $X$ avec 2 taches symétriques caractéristiques (fig. 112 et. 113)

- Sternite X sans les 2 taches sombres caractéristiques

7. - Plaque ventrale en vue frontale avec marques dentelées à l'angle situé entre le bord ventral et le bord latéral ; cornes dorsales petites (fig. 86)

carthusiense
- Plaque ventrale avec marques dentelées moins marquées et cornes dorsales plus grandes (fig. 87) quasidecolletum

8. - Xème sternite clairement distinct en 2 parties, une plus restreinte que l'autre

- Xème sternite plus ou moins carré sans distinction en 2 parties (fig. 111)

carpathicum

9. - Soies de la partie médiane du corps de la plaque ventrale insérées chacune sur une excroissance en forme de mamelon ; Xème sternite divisé en 2 par une constriction médiane en 2 parties environ de même grandeur (fig. 110)

cryophilum

- Soies de la portion médiane ventrale du corps de la plaque ventrale sans structure basale particulière ; Xème sternite en forme de " $\mathrm{T}$ n (fig. 109)

brevidens

\subsubsection{Imagos femelles}

1. - Mandibule dentelé d'un seul côté ; pattes jaunes et noires gr. ruficorne 2

- Mandibule large, dentelé des 2 côtés (fig. 14) , pattes brunes ou noires ......... gr. vernum 3

2. - Gonapophyses distinctement séparées du 8ème sternite, avec bord interne concave

(fig. 118)

angustitarse

- Gonapophyses apparemment fusionnées avec le 8 ème sternite; bord interne convexe (fig. 119)

latigonium

3. - Furca avec expansion plus ou moins développée à l'extrémité des rames médianes (fig. 142a) . 4

- Furca sans expansion à l'extrémité des rames médianes (fig. 142b)

4. - Crypte sensorielle du palpe maxillaire petite, ronde, environ $1 / 2$ de l'article du palpe la contenant (fig. 131) ; cerques grands, caractéristiques (fig. 141)

vernum

- Crypte sensorielle grande, ovoïde, occupant les $2 / 3$ de l'article qui la contient (fig. 132); cerques de forme et proportions diverses

5. - Front de la même largeur au sommet et à la base carthusiense

- Front plus large au sommet qu'à la base quasidecolletum

6. - Cerques 3 fois plus larges que hauts bertrandi

- Cerques moins de 3 fois plus larges que hauts

7. - Dernier article du palpe une fois et demi plus long que le précéđent

(fig. 133) cryophilum + armoricanum

- Dernier article du palpe plus du double en longueur que le précédent $(\mathrm{fig} .132) \ldots \ldots \ldots \ldots \ldots \ldots \ldots$ 
8. - Gonapophyses ne dépassant pas le point de bifurcation de rames latérales de la furca (fig. 120)

brevidens

- Gonapophyses dépassant, jusqu'à plus de la moitié le point de bifurcation des branches latérales de la furca (fig. 121)

carpathicum

\subsection{Sous-genre Obuchovia}

Larve : antenne courte, à taches claires et sombres ; submentum très élargi postérieurement avec dent médiane pointue et soies latérales longues, robustes et disposées en ligne ; disque adhesif avec nombreuses files de crochets. Nymphe : cocon en forme de botte, à texture compacte et talon très prononcé (fig. 72b) ; 6 filaments respiratoires courts et arrondis à leur extrémité distale (fig. 72a). Mâle : styles très longs ; $\mathbf{4}$ fois plus que la hauteur du coxite (fig. 108) ; plaque ventrale caractéristique (fig. 89 ). Femelle : Gonapophyses formées par deux lobes fortement pointus (fig. 122)

* Cascades ; courants violents.

\subsection{Sous-genre Rubzovia}

Larve : corps entier de couleur très pâle, marques céphaliques noires ; échancrure ventrale petite, mais bien délimitée, formant une légère entaille.

Nymphe : deux filaments branchiaux très dilatés (fig. 69). Mâle : styles en forme de cônes, plus courts que les coxites ; plaque ventrale avec carêne médiane large et pourvue de soies (fig. 90b).

Femelle : Gonapophyses avec lobes très arrondis ; spermathèque avec marques polygonales

lamachi

* Ruisselets de sources ou sources, de faible luminosité.

\subsection{Sous-genre Simulium}

4.6.1. Larves

1. - Echancrure ventrale de la tête rejoignant toujours la bordure postérieure du submentum; bords de l'échancrure ventrale bien distincts au point de rencontre avec le bord postérieur du submentum (fig. 26)

- Echancrure ventrale de la tête ne rejoignant généralement pas le bord postérieur du submentum ; quand elle le rejoint, les bords latéraux sont presque en contact avec le point de rencontre du bord postérieur du submentum

2. - Soies filtrantes de la prémandibule d'un seul type ; processus tp de la mandibule avec une seule dent bien évidente suivie d'une petite encoche

degrangei

* Hyporhytron; piémont de petites rivières de montagne.
- Soies filtrantes de la prémandibule de 2 types (soies grandes, épaisses, et intermédiaires plus minces) ; processus tp de la mandibule avec 2 dents de différentes longueurs

3. - Frontoclypeus sombre sur les bords, clair au centre, avec empreintes musculaires latérales indistinctes ; échancrure ventrale (fig. 27)

argenteostriatum

* Torrents de montagne; rhithron.

- Frontoclypeus clair, avec taches médianes et latérales visibles ; échancrure ventrale (fig. 28)

hispaniola

* Moyennes et grandes rivières de piémont.

4. - Echancrure ventrale plus ou moins arrondie et peu profonde

- Echancrure ventrale plus profonde et pointue 7

5. - Distance entre la bordure et l'échancrure ventrale et le bors postérieur du submentum supérieure à la hauteur du submentum

- Distance entre le bord postérieur du submentum et l'échancrure ventrale égale à la hauteur du submentum

intermedium

* Rivières de plaine et piémont.

6. - Echancrure ventrale peu profonde, plus ou moins arrondie (fig. 3a) ; marques antéro-latérales distinctes des autres marques pigmentées du frontoclypeus, marque postéro-médiane étroite : sa hauteur est égale à 3-5 fois sa largeur maximale (complexe d'espèces)

ornatum

* Rivières et ruisseaux lents avec végétation.

- Marques antéro-latérales du frontoclypeus indistinctes incorporées en partie dans l'aire pigmentée des bords latéraux ; marque postéro-médiane large : sa hauteur est inférieure à 2 fois sa largeur maximale (fig. 29a); échancrure ventrale peu profonde avec bord distinct, plat ou en V inversé (fig. 29b) ; épines écailleuses noires autour des branchies rectales trifasciatum

* Relativement rare; rivière de piémont.

7. - Echancrure ventrale très profonde ; distance de la partie antérieure de l'échancrure ventrale à la par. tie postérieure du submentum inférieure à 2 fois la profondeur de l'échancrure ventrale

8

- Echancrure ventrale moins profonde ; distance de la partic antéricure de l'échancrure ventrale à la partie postérieure du submentum supérieure à 2 fois la profondeur de l'échancrure ventrale ....... 11

8. - Echancrure ventrale bien visible, de forme lancéolée (fig. 35b) noelleri

* Déversoirs et dénivellations ; biefs.

- Echancrure ventrale aux limites peu visibles 
9. - Echancrure ventrale rejoint presque la bordure postérieure du submentum (fig. 32) reptans

* Grandes rivières de piémont ; hyporhithron.

- Echancrure ventrale ne rejoint pas la bordure postérieure du submentum

10. - Frontoclypeus très clair ; empreintes musculaires très peu visibles; sourcils bien visibles sur les points visuels

tuberosum

* Torrents ; assez rare.

- Frontoclypeus avec marque centrale nette en forme de lettre " $\mathrm{H}$ 》(fig. 30 ) posticatum

11. - Couleur du corps et capsule céphalique jaune pâle xanthinum

* Ruisseaux calcaires.

- Coulesur de la capsule céphalique de tẹinte foncée

12. - Dent médiane de la bordure antérieure du submentum plus longue et plus robuste que les latérales et intermédiaires ( $\mathrm{ig} .42 \mathrm{~b}$ ) ; aire effilée de cuticule pâle entre le sommet de l'échancrure ventrale et la base du submentum ; échancrure ventrale grande (fig. 31)

variegatum

* Très abondante dans le piérnont et rivières à substrat rocheux.

- Dent médiane de la bordure antérieure du submentum de même longueur que les dents latérales et intermédiaires (fig. 42c) ; échancrure ventrale moins profonde (fig. 34)

13. - Frontoclypeus avec empreintes musculaires plus ou moins distinctes, en forme de trident $\quad 14$

- Frontoclypeus avec impressions musculaires indistinctes ; zone sombre à la bordure postérieure $\mathrm{du}$ frontoclypeus (fig. 33a)

maximum

* Torrents de montagne.

14. - Disque adhésif avec environ 73 files de crochets ; échancrure ventrale pentagonale (fig. 34)

argyreatum

* Abondante dans les torrents de montagne ; sténotherme d'eau froide.

- Disque adhésif avec 90 rangées de crochets; échancrure ventrale plus triangulaire (fig. 33b)

monticola

* Fréquente dans les torrents de montagne ; sténotherme d'eau froide.

\subsubsection{Nymphes}

1. - Cocon avec processus médian et latéraux à son bord anterieur (fig. 66)

- Cocon sans processus médian et latéraux à son bord antérieur (fig. 62)
3. - Cocon avec pointe médiane dorsale et deux ouvertures distinctes latérales (fig. 64) ; tronc basal de l'organe respiratoire bien distinct (fig. 47) argenteostriatum

- Cocon sans pointe médiane dorsale, avec ouver tures latérales indistinctes ou de texture lâche (fig. 65 ) ; tronc commun pratiquement sessile (fig. 48)

hispaniola

4. - Cocon à texture compacte, sans ouvertures ; 6 filaments respiratoires

5

- Cocon à texture plus ou moins lâche, avec fenêtres latérales plus ou moins grandes; 6 ou 8 filaments respiratoires

8

5. - 6 filaments bien séparées entre eux ; cocon petit

- 6 filaments accolés

fuberosum

6. - Filaments respiratoires convexes dorsalement (fig. 49) ; tubercules petits, lisses argyreatum

- Filaments respiratoires concaves dorsalement (fig. 50)

7. - La paire de filaments ventraux provient d'un tronc commun bien distinct (fig. 51) maximum

- La paire de filaments ventraux provient d'un tronc commun sessile (fig. 50) monticola

8. - 6 filaments respiratoires ...................... 9

- 8 filaments respiratoires ....................... 11

9. - A la base de l'organe respiratoire, tégument du thorax avec gros renflement caractéristique ou « patagium " (fig. 56) variegatum

- Pas de "patagium » à la base des filaments respiratoires

10

10. - 6 filaments dans un même plan, de couleur claire posticatum

- 6 filaments dans plusieurs plans et de couleur brun-noir (fig. 70a) ; cocon (fig. 70b) xanthinum

11. - Grand cocon de structure lâche ; 8 filaments respiratoires $(3+3+2)$ disposés dans des plans différents (fig. 67a et b)

noelleri

- Cocon à texture lâche surtout dans la partie antérieure ; les 8 filaments sont disposés dans le même plan

12. - Partie antérieure du cocon avec grandes ouvertures latérales (fig. $71 \mathrm{~b}$ ) ; petite espèce ; filaments branchiaux orientés parallèlement à leur base (fig. 71a)

reptans

- Partie antérieure du cocon sans grandes ouvertures latérales; avec petites ouvertures au point de rencontre entre les bords antérieur et latéral ; filaments branchiaux disposés en arrondi (fig. 5)

13. - Thorax avec tubercules lisses

14

- Thorax avec tubercules épineux

trifasciatum 
14. - Trones communs des filaments respiratoires sessiles ou peu développés; cocon à texture lâche (dans les Pyrénées) (fig. 5) intermedium

- Troncs communs des filaments branchiaux plus ou moins développés ; cocon à texture plus compacte que l'espèce précédente

ornatum

\subsubsection{Imagos mâles}

1. - Plaque ventrale en vue ventrale, plate (fig. 9) ; en vue latérale, sans dentelure sur le bord distal

- Plaque ventrale, en vue ventrale, étroite ; en vue latérale avec dentelure (plusieurs dents) sur le bord distal en forme d'éperon (fig. 95 et 96)

4

2. - Plaque ventrale caractéristique, avec 2 expansions latérales amples en forme d'aile ( $\mathrm{fig}$. 9a) . 3

- Plaque ventrale simple, avec corps quadrangulaire et bras dorsaux robustes (fig. 9b) degrangei

3. - Style à bords légèrement concaves (fig. 9a) argenteosiriatum

- Style à bords moins concaves, presque droits hispaniole

4. - Plaque ventrale vue de front, en forme de bouteille, avec bras en « $V$ » (fig. 91a) ......... 5

- Plaque ventrale vue de front, de forme quadrangulaire (fig. 96a)

5. - Corps de la plaque ventrale vue de front élargi en bouteille, 4 fois plus large que le diamètre des bras (fig. 91a) ; mesonotum vu de front, avec taches argentées reliées à 2 stries plus ou moins amples

- Corps de la plaque ventrale vue de front, 2 fois à 2 fois et demi le diamètre des bras (fig. 95a); mesonotum vu de front avec taches argentées non reliées à une strie, avec petites soies jaune doré

6. - Plaque ventrale, en vue latérale (fig. 91b), avec bord distal épineux approximativement $1 / 2$ circulaire et processus ventral nasiforme à extrémité légèrement retournée

ornatum

- Plaque ventrale en vue latérale (fig. 92) avec bord épineux dorsal plus étroit

intermedium

- Plaque latérale en vue latérale (fig. 93b) avec bord distal épineux descendant verticalement à la base du processus ventral nasiforme large à extrémité arrondie

trifasciatum

7. - Longueur des bras de la plaque ventrale vue de front, légèrement inférieure ou égale à la hauteur du corps de la plaque ventrale ; processus nasiforme (en vue latérale) en forme de triangle équilatéral et bord distal épineux très étroit $(f i g .95 \mathrm{~b}$ )
- Longueur des bras de la plaque ventrale légèrement supérieur à la hauteur du corps de la plaque ventrale (vue frontale) ; processus nasiforme très petit, environ le tiers de la hauteur du bord épineux qui, inversement, est très ample et quadrangulaire (fig. 95c)

8. - Sclérite médian allongé, triangulaire (fig. 95e) ; mesonotum à ornementation noire sur fond jaune; crochets des paramères nombreux et de taille sensiblement égale xanthinum

- Sclérite médian étroit, divisé en deux distalement (fig. 95d); mesonotum avec différentes taches argentées sur fond noir (fig. 138); crochets des paramères (9-10) avec dimensions diminuant progressivement vers l'avant

variegatum

9. - Style très petit (fig. 101) ; basitarse I relativement mince, 6 fois plus long que large argyreatum

- Style de moyenne ou grande dimension ; basitarse I un peu plus large, environ 5 fois plus long que large

10. - Style de grande dimension, plus étroit à l'extrémité qu'à la base, avec épine terminale située près de l'extrémité apicale (fig. 102) maximum

- Style de moyenne dimension, environ de même largeur sur toute son extension ; épine terminale éloignée de l'extrémité apicale (fig. 103)

monticola

11. - Style caractéristique avec une protubérance basale (fig. 107)

- Style sans protubérance à la base 12

12. - Style sans protubérance basale bien apparente. grande, munie de petites épines (fig. 107)

uberosum

- Style avec protubérance peu apparente posticartum

13. - Plaque ventrale, en vue latérale, avec 9-10 den[4 (fig. 94) noelleri

- Plaque ventrale en vue latérale, avec 1 ou 2 dents (fig. 96b) reptans

\subsubsection{Imagos femelles}

1. - Mesonotum parcouru sur toute sa longueur par 3 stries argentées, une médiane mince et 2 latérales plus amples (fig. 136)

- Mesonotum avec une striature argentée uniquement dans sa partie antérieure médiane ; latéralement, taches argentées limitées à la partie antérolatérale ou sans taches

2. - Furca avec tige médiane net tement dilatée distalement

- Furca avec tige médiane non dilatée distalement degrangei 
3. - Gonapophyses légèrement pointues dans le sens postérieur argenteostriatum

- Gonapophyses à bord droit hispaniola

4. - Griffe avec petite dent basale (fig. 13a) ... 5

- Griffe sans petite dent basale 10

5. - Sur le mesonotum, entre les 2 taches argentées latérales pas de tache médiane plus ou moins triangulaire

- Présence d'une grande bande médiane argentée entre les 2 taches argentées latérales sur le mesonotum (fig. 139)

6. - Sur le mesonotum, entre la tache argentée médiane et les 2 latérales, présence d'une grande tache sombre intermédiaire $($ fig. 134)

- Sur le mesonotum, entre la tache argentée médiane et les 2 latérales, une strie très mince, sombre, intermédiaire (fig. 138)

variegatum

7. - Front à coloration foncée ou grise, jamais brilIante

- Front à coloration noire brillante intermedium

8. - Espace entre les 2 gonapophyses plutôt rétréci ; Bt 1 pas trè élargi et pas très aplati dorsoventralement

irifasciatum

- Espace entre les 2 gonapophyses plutôt ample (fig. 123) ; Bt I fortement aplati et élargi dorsoventralement

ornotum

9. - Gonapophyses très grandes avec expansions latérales rapprochées ; branche médiane de la furca environ $11 / 2$ plus longue que chacune des 2 branches latérales ; sclérites bien prononcés (fig. 124); front noir brillant

argyreatum

- Gonapophyses grandes rapprochèes côté proximal, courbure interne concave, maximale à la $1 / 2$ hauteur, bord reste éloigné et droit côté apical (fig. 126) maximum

- Gonapophyses petites triangulaires; rame médiane de la furca seulement un peu plus lon. gue que chacune des branches latérales (fig. 125)

monticola

10. - Lisières internes des gonapophyses légèrement recourbées entre elles, étroitement rapprochées sur la ligne médiane

- Bord interne de la gonapophyse non recourbé et nettement éloigné de la ligne médiane (fig. 128); mesonotum avec 2 aires argentées triangulaires en vue antérieure (fig. 135)

reptans

11. - Bord interne de la gonapophyse lëgèrement convexe ou droit et en contact ou presque sur la ligne médiane (fig. 129) ; mesonot um sans taches, gris en vue antérieure ; front et clypeus noir brillant

tuberosum
- Bord interne de la gonapophyse légèrement concave (fig. 130) ; front et clypeus gris noir mat noelleri

- Bord interne presque droit légèrement concave ; bord apical perpendiculaire au bord interne ; front gris noir brillant

posticatum

\subsection{Sous-genre Tetisimulium}

Larve : dents du submentum très prononcées, les latérales inclinées vers l'extérieur (fig. 41) ; échancrure ventrale profonde en angle aigu.

Nymphe : cocon à texture lâche avec ouvertures variées au bord antérieur (fig. 15).

Mâle : couleur noire avec taches argentées sur mesonoturn ; basitarse I non dilaté, cylindrique, $7-8$ fois plus long que large.

Femelle : coloration de fond grise avec 3 grandes stries longit udinales noires, (fig. 137) ; basitarse I non dilaté ; griffes très robustes avec dent basale bien visible bezzii * Torrent de montagne en zone de piémont ; une certaine résistance aux matières organiques.

\subsection{Sous-genre Wilhelmia}

Larve : dents du submentum d'égales dimensions $(f i g$. 42) ; échancrure ventrale profonde, ogivale ; disque postérieur avec nombreuses files de crochets.

Nymphe : cocon de texture compacte et en forme de chaussure (fig. 15 et 68 ).

Mâle : membrane pleurale avec nombreuses soies ; basitarse étroit, environ la moitié de la largeur maximale du tibia ; plaque ventrale (fig. 82).

Femelle : gonapophyses avec expansions en forme de ruban (fig. 127) lineatum

* Grands fleuves de plaine ; ici, biotope particulier : ruisseaux lents, riches en matières organiques et soumis à des températures estivales élevées.

\section{Remerciements}

L'auteur remercie cordialement Monsieur le Professeur Giudicelli, dont les critiques ont permis une amélioration de ce manuscrit.

\section{Travaux cités}

Crosskey (R.W.). 1987. - An annotated checklist of the world blackflies (Diptera : Simuliidae). In : Blackflies, Ecology, population management, and annotated world list. Kim (K.C.) \& Merritt (R.W.) ed. Pennsylvania state university U.S.A.

Davies (L.). 1968. - A key to the british species of Simuliidae (Diptera) in the larval, pupal and adult stages. Freshwater Biological, Association Scientific Publication, 24 : $1-125$. 
Giudicelli (J.). 1968. - Recherches sur le peuplement, l'écologie et la biogéographie d'un réseau hydrographique de la Corse centrale. Thèse de Doctorat ès Sciences Naturelles, Université Aix-Marseille, CNRS A.O. 2478. 437 p.

Grenier (P.). 1953. - Simuliidae de France et d'Afrique du Nord. Encyclopédie Ent., Sér. A, Paris, $29: 1-170$.

Jensen (J.). 1984. - A revision of the Danish black-flies (Diptera : Simuliidac), with keys to the larval and pupal stages. Natura Jutlandico, 21 (6) : 69-116.

Knoz (J.). 1965 . - To identification of czechoslovakian blackflies (Diptera, Simuliidae). Folia Fak. Scient. natur. Univers. Purk. (Biol. 2) Brno, 6 (5) : 1-54.

Rivosecchi (L.). 1967. - Simuliidi degli Apennini. Parassirol. Roma, 9 (3) : 129-304.
Rivosecchi (L.). 1978a. - Guide per il riconoscimento delle specie animali delle acque interne italiane. 3. Simuliidi (Diptera Simuliidae) Consiglio nazionale delle ricerche $\mathrm{AQ} / \mathrm{l} / 7: 1-88$. Rivosecchi (L.). 1978b. - Simuliidae Diptera Nematocera. Fauna Italia 13. VIJI. Accademia Nazionale Italia di Entomol, e del Unione Zool. Italiana. Bologna, 533 p.

Vinçon (G.). 1987. - Comparaison de la faune benthique des vallés d'Aure el d'Ossau, en vue de l'élaboration d'une méthodologie de surveillance des cours d'eau de montagne. Thèse de Docteur-Ingénieur, Toulouse, $n^{\circ} 960,381 \mathrm{p}$. 